\title{
Multiple sclerosis Lesion Detection via Machine Learning Algorithm based on converting 3D to 2D MRI images
}

\author{
Mohammad Moghadasi ${ }^{1}$ and Gabor Fazekas ${ }^{2}$
}

\begin{abstract}
In the twenty first century, there have been various scientific discoveries which have helped in addressing some of the fundamental health issues. Specifically, the discovery of machines which are able to assess the internal conditions of individuals has been a significant boost in the medical field. This paper or case study is the continuation of a previous research which aimed to create artificial models using support vector machines (SVM) to classify MS and normal brain MRI images, analyze the effectiveness of these models and their potential to use them in Multiple Sclerosis (MS) diagnosis. In the previous study presented at the Cognitive InfoCommunication (CogInfoCom 2019) conference, we intend to show that $3 D$ images can be converted into 2D and by considering machine learning techniques and SVM tools. The previous paper concluded that SVM is a potential method which can be involved during MS diagnosis, however, in order to confirm this statement more research and other potentially effective methods should be included in the research and need to be tested. First, this study continues the research of SVM used for classification and Cellular Learning Automata (CLA), then it expands the research to other method such as Artificial Neural Networks (ANN) and k-Nearest Neighbor $(\mathrm{k}-\mathrm{NN})$ and then compares the results of these.
\end{abstract}

Keywords- Support Vector Machines (SVM); Cellular Learning Automata (CLA); MS lesions Detection; 3D Images; MRI Images; Simulated Brain Database (SBD); SVM Tools; Machine Learning Techniques;

\section{INTRODUCTION}

CogInfoCom, the research field based on the synergy between info communications and the cognitive sciences, is driven by the continuously entangled landscape in which Information and Communications Technology (ICT) and humans interact and generate intermingled cognitive capabilities [1], [2]. CogInfoCom capitalizes on this intermingled environment and promotes existing synergies creating a more effective combination of engineering and theoretical

\footnotetext{
This work was not supported by any organization.

${ }^{1,2}$ Department of Informatics, University of Debrecen, Debrecen, Hungary

IE-mail: Mohammad.Moghadasi@inf.unideb.hu

2E-mail: Fazekas.Gabor@inf.unideb.hu
}

applications. A primary output of these synergies improved in a way that does not provide just sensory communications but also the way information is stored in the brain. [3], [4], [5], [6].

This study is the continuation of a previous research which aimed to create artificial models using support Vector machines (SVM) to classify MS and normal brain MRI images, analyze the effectiveness of these models and their potential to use them in MS diagnosis. The previous study concluded that SVM is a potential method which can be involved during MS diagnosis, however in order to confirm this statement more research and other potentially effective methods should be included in the research and need to be tested [7], [8].

Multiple sclerosis (MS) a chronic autoimmune neurological disease of the Central Nervous System (CNS) which appears with great variability in its clinical manifestation [9]. MS adjust the morphology and the structure of the brain and can lead to disability in young adults (Loizou et al., 2011) [10], [11], [12]. However, with early recognition and treatment, quality of life can be highly improved and the relapse of MS lesions in the CNS can be experienced (Miller, 2019) [13], [14].

Magnetic Resonance Imaging (MRI) can detect the multifocal lesions in the CNS mainly associated with MS. In the previous research a simulated database of 2D images was used, which were generated from simulated 3D dataset, acquired from Brainweb database [15], [16]. This dataset contains 76 grayscale images classified into four classes, samples with normal brain images, mild MS samples, moderate MS samples and severe MS samples [17], [18], [19].

First, this study continues the research of SVM used for classification and then it expands the research to other method such as artificial neural networks (ANN) and k-nearest neighbor $(\mathrm{k}-\mathrm{NN})$ and then compares the results of these [20], [21], [22].

\section{DATASET}

In the previous study the dataset used for model training and model testing was randomly generated, 70\% (53 images) of the images used for training and 30\% (23 images) for testing and the 
process was repeated 10 times. In this case since the intention is to try more methods and compare them, the same dataset should be used for each test [23], [24], [25]. To achieve this the indices of the test dataset for each run are saved into a file. For each run, this file is read, processed and the samples which indices are contained in the file are used to test the methods, while the samples which indices are not contained in the file are used to train the models [26].

\section{RESULTS OF USING SVM}

SVM is one of the most widely used machine learning method for binary pattern classification. SVM aims to construct a hyperplane set in an infinite dimensional space and find the hyperplane which represents the largest separation (margin) between the binary classes, so the goal is to find the maximummargin hyperplane if it exists (Chao and Horng, 2015) [27], [28], [29], [30].

In the previous study two approaches were used since SVM is a binary classifier, however the current dataset can be divided into four mutually exclusive classes. In order to resolve this, in the previous study One-Against-One (1A1) and One-AgainstAll (1AA) techniques were introduced. The goal of 1AA technique to divide the $\mathrm{N}$ class dataset into $\mathrm{N}$ two-class cases, while 1A1 approach creates a model for each pair of classes so $\mathrm{N}(\mathrm{N}-1) / 2$ models are built. In the previous study each method had an equal vote (Gidudu, Hulley \& Marwala, 2007) [31], [32].

These approaches were reused for the new, fix dataset and to be able to compare the results of the methods the implementation was rerun to build models using the new trainsets and test-sets. For building the model MATLAB ${ }^{\circledR}$ fitcsvm function was used with linear kernel function and with standardized predictor data (Table I.).

The average accuracy of the 1AA model is slightly worse compared to the previous model, however the 1A1 model produced a slightly better result. For the 2AllResult (the accuracy of the 1AA models), the previous average accuracy of the models was 0.77826087 while for 2OneResult (the accuracy of the 1A1 models) models was 0.765217391. so this run, 2OneResult has a slightly better average result and so far, it produced the best results in this run. Another interesting fact that in these runs no sample has been assigned to the 'more_results' flag, so in this case with equal votes for each method resulted in a deterministic result, however the number of no results in the case of 1AA method has a significant grow. In the previous study a rule for a voting system could be determined, which could have been tested in this study. This was not explained in the previous study, but the system would have been the following:

- $\quad$ 1AA method is used for the primary decision.

- if $1 \mathrm{AA}$ resulted in more results, the more severe result should be used.

- if $1 \mathrm{AA}$ resulted in no results, the $1 \mathrm{~A} 1$ result should be used.

- if 1A1 has more results, the more severe should be used.

This rule should be rejected, because of the 1A1 has produced a slightly better result compared to the 1AA method and because no sample got more results in this case, so using such a technique wouldn't improve the overall results. For SVM, a different

TABLE I. SVM RESULTS USING LINEAR KERNEL FUNCTION

\begin{tabular}{|c|c|c|c|c|c|c|c|c|}
\hline$I D$ & $\begin{array}{c}2 A l l \\
\text { Result }\end{array}$ & $\begin{array}{l}\text { 2One } \\
\text { Result }\end{array}$ & $\begin{array}{c}\text { 2AllMore } \\
\text { Result }\end{array}$ & $\begin{array}{c}\text { 2OneMore } \\
\text { Result }\end{array}$ & $\begin{array}{l}2 A l l N o \\
\text { Result }\end{array}$ & $\begin{array}{c}\text { 2OneNo } \\
\text { Result }\end{array}$ & Differences & $\begin{array}{l}S T . \\
D E V\end{array}$ \\
\hline 1 & 0.739130435 & 0.652173913 & 0 & 0 & 6 & 0 & 0.391304 & 0.061488 \\
\hline 2 & 0.739130435 & 0.782608696 & 0 & 0 & 6 & 0 & 0.26087 & 0.030744 \\
\hline 3 & 0.695652174 & 0.739130435 & 0 & 0 & 7 & 0 & 0.304348 & 0.030744 \\
\hline 5 & 0.782608696 & 0.782608696 & 0 & 0 & 5 & 0 & 0.26087 & 0 \\
\hline 6 & 0.739130435 & 0.782608696 & 0 & 0 & 6 & 0 & 0.26087 & 0.030744 \\
\hline 7 & 0.739130435 & 0.739130435 & 0 & 0 & 6 & 0 & 0.26087 & 0 \\
\hline 10 & 0.695652174 & 0.782608696 & 0 & 0 & 7 & 0 & 0.304348 & 0.061488 \\
\hline$A V G$ & 0.743478261 & 0.766304348 & 0 & 0 & 5.9 & 0 & 0.273913 & 0.01614 \\
\hline
\end{tabular}


Multiple sclerosis Lesion Detection via Machine Learning Algorithm

based on converting $3 \mathrm{D}$ to $2 \mathrm{D} \mathrm{MRI}$ images

TABLE II. SVM RESULTS USING RBF KERNEL FUNCTION

\begin{tabular}{|cccccccc|r|}
\hline ID & $\begin{array}{c}\text { 2All } \\
\text { Result }\end{array}$ & $\begin{array}{c}\text { 2One } \\
\text { Result }\end{array}$ & $\begin{array}{c}\text { 2AllMore } \\
\text { Result }\end{array}$ & $\begin{array}{c}\text { 2OneMore } \\
\text { Result }\end{array}$ & $\begin{array}{c}\text { 2AllNo } \\
\text { Result }\end{array}$ & $\begin{array}{c}\text { 2OneNo } \\
\text { Result }\end{array}$ & Differences & $\begin{array}{c}\text { ST. } \\
\text { DEV }\end{array}$ \\
\hline 1 & 0.739130435 & 0.782608696 & 0 & 0 & 6 & 0 & 0.260869565 & 0.030744 \\
\hline 2 & 0.739130435 & 0.782608696 & 0 & 0 & 6 & 0 & 0.260869565 & 0.030744 \\
\hline 3 & 0.695652174 & 0.739130435 & 0 & 0 & 7 & 0 & 0.304347826 & 0.030744 \\
\hline 4 & 0.826086957 & 0.826086957 & 0 & 0 & 4 & 0 & 0.173913043 & 0 \\
\hline 5 & 0.782608696 & 0.826086957 & 0 & 0 & 5 & 0 & 0.217391304 & 0.030744 \\
\hline 6 & 0.739130435 & 0.739130435 & 0 & 0 & 6 & 0 & 0.260869565 & 0 \\
\hline 7 & 0.739130435 & 0.739130435 & 0 & 0 & 6 & 0 & 0.260869565 & 0 \\
\hline 8 & 0.695652174 & 0.782608696 & 0 & 0 & 7 & 0 & 0.304347826 & 0.061488 \\
\hline 9 & 0.782608696 & 0.826086957 & 0 & 0 & 5 & 0 & 0.217391304 & 0.030744 \\
\hline 10 & 0.695652174 & 0.739130435 & 0 & 0 & 7 & 0 & 0.304347826 & 0.030744 \\
\hline $\boldsymbol{A V G}$ & $\mathbf{0 . 7 4 3 4 7 8 2 6 1}$ & $\mathbf{0 . 7 7 8 2 6 0 8 7 0}$ & $\mathbf{0}$ & $\mathbf{0}$ & $\mathbf{5 . 9}$ & $\mathbf{0}$ & $\mathbf{0 . 2 5 6 5 2 1 7 3 9}$ & 0.024595 \\
\hline
\end{tabular}

kernel function can be tested to examine the method efficiency [33]. This kernel function is the radial basis function (rbf) and the result can be seen in (Table II).

For the 1AA method the rbf kernel function produced exactly the same results compared to the linear kernel function, however in the case of $1 \mathrm{~A} 1$ the results are slightly better for these tests. These runs also did not produce more results for any of the elements of the datasets.

\section{RESUlts USING NEURAL NeTWORKS}

A neural network $(\mathrm{NN})$ is built from simple, connected processors called neurons which form a sequence of real-valued activations. Special type of neurons, called input neurons are activated through the environment, other neurons are activated through the connected previously activated, weighted neurons. The learning process of a neural network is finding the optimal weights to be able to perform the desired results (Table III). The construction of a network depends on the problem and how the neurons are connected (Schmidhuber, 2015) [34].

MATLAB ${ }^{\circledR}$ provides easy to use, flexible tools to create, test and analyze neural networks. In this study a simple artificial neural network (for the exact parameters see Appendix A), with 15 layers and 10 max epochs were trained using the sample train- and test datasets which were used to build the SVM models.

The average accuracy of the NN network is significantly worse compared to the SVM models, however the 9th NN test produced an especially low accuracy. Here the model supposedly did not conver-gate, so the whole test was repeated using 50 max epochs.

This modification significantly improved the results of the NN and for test 2 and test 9 the model reached $100 \%$
TABLE III. ACCURACY OF NN USING 10 AND 50 MAXIMUM EPOCHS

\begin{tabular}{|cc|c|}
\hline ID & $\begin{array}{c}\text { Accuracy } \\
\text { (10 epochs) }\end{array}$ & $\begin{array}{c}\text { Accuracy } \\
\text { (50 epochs) }\end{array}$ \\
\hline 1 & 0.82608696 & 0.95652174 \\
\hline 2 & 0.82608696 & 1 \\
\hline 3 & 0.60869565 & 0.82608696 \\
\hline 4 & 0.69565217 & 0.86956522 \\
\hline 5 & 0.7826087 & 0.95652174 \\
\hline 6 & 0.60869565 & 0.7826087 \\
\hline 7 & 0.86956522 & 0.86956522 \\
\hline 8 & 0.69565217 & 0.82608696 \\
\hline 9 & 0.26086957 & 1 \\
\hline 10 & 0.82608696 & 0.95652174 \\
\hline $\boldsymbol{A V G}$ & $\mathbf{0 . 7}$ & $\mathbf{0 . 9 0 4 3 4 7 8 3}$ \\
\hline
\end{tabular}

accuracy. However, this could imply that the models are overtrained, which means that they produce the desired results for this sample, however for different samples they would not perform this well. Cross validation technique is used to avoid this situation, however more independent tests with additional samples would be needed to confirm or reject the case of overtraining [35], [36], [37].

So far NN reached the best average accuracy and supposedly the $\mathrm{NN}$ result would be improved if the number of epochs would be increased. In case of neural networks, the challenge is to determine the structure of the network, for instance should it be feed forward or recurrent and other important structural decisions also highly effect the desired behavior of the networks, for instance the number of layers, the type of this layers, the number of neurons in each layer etc.

For k-NN MATLAB ${ }^{\circledR}$ provides a complex framework to train the models and use the models for prediction [38]. 
The models are created using the "fitcknn" function which provides a highly parameterizable, flexible solution for model training. In the study, several parameters are used, the number of neighbors and the distance metrics were called "Minkowski" distance and Euclidean distance were used.

Minkowski distance was tested with $k=3 . .7$ values. Compared to the SVM and the NN, the average accuracy produced by k-NN is the worst. The best result was reached with $k=4$, however a tendency can be observed, with increasing $\mathrm{k}$ the average accuracy was decreased [28], [39], [40], [41].

\section{RESULTS USING K-NN}

It is reasonable to expect those samples which are close using an appropriate metric can be classified into the same class and it is also applicable to assume, that one unclassified observation can be classified to the class where its closest neighbors are classified (Dudani, 1976) [21]. The simplest knearest neighbor (k-NN) computes the distance (or similarity) between the sample and the values in the training set (Table IV)., restricts the maximum number of neighbors to $\mathrm{k}$ training samples and assign the class to the sample which the majority of the k neighbors belong (Grudzinski, 2008) [42], [43], [44].

The k-NN test was repeated with $k=1 . .7$ and using Euclidean distance as the distance metrics. The results can be seen in (Table V).

Using the Euclidean distance for building the model did not improve the average accuracy for $k=3 . .7$, however the average accuracy for $k=1$ is significantly better compared to other $\mathrm{k}$ values. This can be due to the variety which can be experienced in MS lesions in the dataset, however more analysis would be needed to prove that.

TABLE IV. K-NN RESUlTS USING MINKOWSKI DISTANCE WITH $k=3 . .7$

\begin{tabular}{|cccccc|}
\hline ID & Accuracy3 & Accuracy4 & Accuracy5 & Accuracy6 & Accuracy \\
\hline 1 & 0.695652 & 0.695652 & 0.695652 & 0.652174 & 0.652174 \\
\hline 2 & 0.565217 & 0.565217 & 0.565217 & 0.521739 & 0.565217 \\
\hline 3 & 0.478261 & 0.478261 & 0.478261 & 0.521739 & 0.434783 \\
\hline 4 & 0.695652 & 0.869565 & 0.73913 & 0.695652 & 0.478261 \\
\hline 5 & 0.695652 & 0.521739 & 0.521739 & 0.478261 & 0.521739 \\
\hline 6 & 0.565217 & 0.565217 & 0.521739 & 0.478261 & 0.478261 \\
\hline 7 & 0.521739 & 0.521739 & 0.521739 & 0.478261 & 0.478261 \\
\hline 8 & 0.608696 & 0.652174 & 0.565217 & 0.565217 & 0.565217 \\
\hline 9 & 0.608696 & 0.608696 & 0.565217 & 0.521739 & 0.26087 \\
\hline 10 & 0.434783 & 0.478261 & 0.434783 & 0.478261 & 0.521739 \\
\hline $\boldsymbol{A} \boldsymbol{V G}$ & $\mathbf{0 . 5 8 6 9 5 7}$ & $\mathbf{0 . 5 9 5 6 5 2}$ & $\mathbf{0 . 5 6 0 8 7}$ & $\mathbf{0 . 5 3 9 1 3}$ & $\mathbf{0 . 4 9 5 6 5 2}$ \\
\hline
\end{tabular}

TABLE V. K-NN RESUltS USING EUCLIDEAN DISTANCE WITH K=1..7

\begin{tabular}{|cccccccc|}
\hline ID & Accuracy1 & Accuracy2 & Accuracy3 & Accuracy4 & Accuracy5 & Accuracy6 & Accuracy7 \\
\hline 1 & 0.826087 & 0.695652 & 0.695652 & 0.695652 & 0.695652 & 0.652174 & 0.652174 \\
\hline 2 & 0.782609 & 0.652174 & 0.565217 & 0.565217 & 0.565217 & 0.521739 & 0.565217 \\
\hline 3 & 0.782609 & 0.565217 & 0.478261 & 0.478261 & 0.478261 & 0.521739 & 0.434783 \\
\hline 4 & 0.913043 & 0.782609 & 0.695652 & 0.869565 & 0.73913 & 0.695652 & 0.478261 \\
\hline 5 & 0.826087 & 0.695652 & 0.695652 & 0.521739 & 0.521739 & 0.478261 & 0.521739 \\
\hline 6 & 0.826087 & 0.565217 & 0.565217 & 0.565217 & 0.521739 & 0.478261 & 0.478261 \\
\hline 7 & 0.782609 & 0.521739 & 0.521739 & 0.521739 & 0.521739 & 0.478261 & 0.478261 \\
\hline 8 & 0.913043 & 0.695652 & 0.608696 & 0.652174 & 0.565217 & 0.565217 & 0.565217 \\
\hline 9 & 0.826087 & 0.608696 & 0.608696 & 0.608696 & 0.565217 & 0.521739 & 0.26087 \\
\hline 10 & 0.73913 & 0.478261 & 0.434783 & 0.478261 & 0.434783 & 0.478261 & 0.521739 \\
\hline $\boldsymbol{A V G}$ & $\mathbf{0 . 8 2 1 7 3 9}$ & $\mathbf{0 . 6 2 6 0 8 7}$ & $\mathbf{0 . 5 8 6 9 5 7}$ & $\mathbf{0 . 5 9 5 6 5 2}$ & $\mathbf{0 . 5 6 0 8 7}$ & $\mathbf{0 . 5 3 9 1 3}$ & $\mathbf{0 . 4 9 5 6 5 2}$ \\
\hline
\end{tabular}




\section{COMPARING THE RESULTS}

The best average accuracy was produced by NN however that is not the only tool to compare results. In this case, the ratio of fake positive and fake negative values also can be counted. In this case the dataset contains four classes, however it can be simplified by assuming that normal is negative and MS effected samples are positive. Therefore, fake positive is, when the classifier assigned a positive class to the sample, however it is actually negative and fake negative is a sample which the classifier assigned a negative value, but it is actually positive. Using this assignment differences between the severity of MS do not counted, just the difference between normal and MS samples and the goal is to minimize these values. The below table (Table VI.) contains the average number of fake positive and fake negative values for every method tested in this study.

$\mathrm{NN}$ using 50 maximum epochs managed to minimize the average number of fake positive results and $\mathrm{k}-\mathrm{NN}$ using Euclidean distance metrics with $k=1$ and all SVM models also reached good average results. In case of fake negative, 1AA SVM models managed to minimize the average values with zero fake negative results in the samples $1 \mathrm{~A} 1 \mathrm{SVM}$ and $\mathrm{NN}$ with epoch 50 .

TABLE VI. AVERAGE NUMBER OF FAKE POSITIVE AND FAKE NEGATIVE RESULT BY METHOD

\begin{tabular}{|c|c|c|}
\hline Method & Fake positive & Fake negative \\
\hline 1AA SVM linear & 1.5 & 0 \\
\hline $1 A 1$ SVM linear & 1.5 & 0.8 \\
\hline $1 A A S V M r b f$ & 1.5 & 0 \\
\hline $1 A 1 S V M r b f$ & 1.5 & 0.9 \\
\hline NN epoch 10 & 2.2 & 3 \\
\hline NN epoch 50 & 1 & 1 \\
\hline$k-N N$ Euclidean $k=1$ & 1.1 & 0.7 \\
\hline$k-N N$ Euclidean $k=2$ & 3 & 1 \\
\hline$k-N N$ Euclidean $k=3$ & 3.1 & 0.9 \\
\hline$k-N N$ Euclidean $k=4$ & 3.1 & 1.2 \\
\hline$k-N N$ Euclidean $k=5$ & 2.9 & 1.7 \\
\hline$k-N N$ Euclidean $k=6$ & 3.2 & 1.3 \\
\hline$k-N N$ Euclidean $k=7$ & 3.3 & 1.5 \\
\hline$k-N N$ Minkowski $k=3$ & 3.1 & 0.9 \\
\hline$k-N N$ Minkowski $k=4$ & 3.1 & 1.2 \\
\hline$k-N N$ Minkowski $k=5$ & 2.9 & 1.7 \\
\hline$k-N N$ Minkowski $k=6$ & 3.2 & 1.3 \\
\hline$k-N N$ Minkowski $k=7$ & 3.3 & 1.5 \\
\hline
\end{tabular}

\section{CONCLUSION}

SVM is a useful tool for MS disease diagnosis process. In order to be able to make more appropriate and satisfactory assumptions, more tests are required. Brainweb dataset is a useful source of data to generate images with different parameters, which can change the parameters such as brightness, contrast and etc. of the images. Tests with SVM, NN and k-NN proved that with the current results $\mathrm{NN}$ is the method which should be widely investigated, however SVM also should not be dropped because of it can minimize the number of fake negative values. For further investigation a new dataset could be built using different simulation parameters. Even artificial neural networks for the 3D simulations can be built if the proper hardware resources are available, but naturally the best dataset would be the data of real MS patients. The current idea is to focus on neural networks, however SVM should be used to confirm the results of $\mathrm{NN}$.

\section{REFERENCES}

[1]. P. Baranyi, A. Csapo and G. Sallai., Cognitive Infocommunications (CogInfoCom), Springer International Publishing, Switzerland, 2015, p. 191, ISBN: 978-3-319-19607-7, Available: http://www.springer.com/us/ book/9783319196077\#aboutBook, DoI: 10.1007/978-3-319-19608-4

[2]. Csapo A, Baranyi P. "A conceptual framework for the design of audio based cognitive infocommunication channels", 2012 Recent advances in intelligent engineering systems. Studies in computational intelligence, vol 368. Springer, Berlin/Heidelberg, pp 261-281. DoI: 10.1007/978-3-642-23229-9_12

[3]. Mousavi, S., Fazekas, G.:“A Novel Algorithm for Load Balancing using HBA and ACO in Cloud Computing Environment", International Journal of Computer Science and Information Security (IJCSIS), 2016, 14(6), pp.48-52. ISSN 1947-5500

[4]. G. Bujdosó, O. C. Novac and T. Szimkovics, "Developing cognitive processes for improving inventive thinking in system development using a collaborative virtual reality system," 2017 8th IEEE International Conference on Cognitive Infocommunications (CogInfoCom), Debrecen, 2017, pp. 000079-000084. Dor: $10.1109 / \operatorname{Cog}$ InfoCom.2017.8268220

[5]. L. Gazdi, K. D. Pomazi, B. Radostyan, B. Forstner, L. Szegletes: "Experimenting with classifiers in biofeedback-based mental effort measurement", 2016 7th IEEE International Conference on Cognitive Infocommunications (CogInfoCom), Wroclaw, 2016. DoI: 10.1109/CogInfoCom.2016.7804571

[6]. Zhang, Yudong, Siyuan Lu, Xingxing Zhou, Ming Yang, Lenan Wu, Bin Liu, Preetha Phillips, and Shuihua Wang.: "Comparison of Machine Learning Methods for Stationary Wavelet Entropy-Based Multiple Sclerosis Detection: Decision Tree, k-Nearest Neighbors, and Support Vector Machine.” SIMULATION 92, no. 9 (September 2016): 861-71. DOI: $10.1177 / 0037549716666962$

[7]. Mohammad Moghadasi and Dr. Gabor Fazekas, "An Automatic Multiple Sclerosis Lesion Segmentation Approach based on Cellular Learning Automata" International Journal of Advanced Computer Science and Applications(IJACSA), 10(7), 2019. DOI: 10.14569/IJACSA.2019.0100726

[8]. Coupland AP, Thapar A, Qureshi MI, Jenkins H, Davies AH.: "The definition of stroke" Journal of the Royal Society of Medicine. 2017 Jan; 110(1):9-12. DOI: 10.1177/0141076816680121 
[9]. Loizou, C.P., Murray, V., Pattichis, M.S., Seimenis, I., Pantziaris, M., Pattichis, C.S.: "Multiscale amplitude-modulation frequencymodulation (AMFM) texture analysis of multiple sclerosis in brain MRI images" 2011 IEEE Transactions on Information Technology in Biomedicine, 2011 ; Vol. 15, No. 1, 5624633, pp. 119-129., DoI: $10.1109 /$ TITB.2010.2091279

[10]. Kamathe, Rupali S. and Kalyani R. Joshi.: "A novel method based on independent component analysis for brain MR image tissue classification into CSF, WM and GM for atrophy detection in Alzheimer's disease." Biomed. Signal Process. Control. 40 (2018): 41-48. DOI: 10.1016/j.bspc.2017.09.005

[11]. Zarei S, Maldonado I, Franqui-Dominguez L, et al. Impact of delayed treatment on exacerbations of multiple sclerosis among Puerto Rican patients. Surgical Neurology International. 2019;10:200. DOI: 10.25259/SNI_252_2019.

[12]. Abbas Talimian, Péter Béda: "Dynamic Stability of a Thin Plate Subjected to Bi-Axial Edged Loads", Acta Polytechnica Hungarica, 2018, 15(2). DoI: 10.12700/APH.15.1.2018.2.7

[13]. C.A. Cocosco, V. Kollokian, R.K.-S. Kwan, A.C. Evans: "BrainWeb: Online Interface to a 3D MRI Simulated Brain Database", NeuroImage, vol.5, no.4, part 2/4, S425, 1997 -- Proceedings of 3-rd International Conference on Functional Mapping of the Human Brain, Copenhagen, May 1997. abstract available in [html], [pdf $(500 \mathrm{~Kb})]$, or [gnuzip-ed postscript $(500 \mathrm{~Kb})]$. poster available in [pdf $(1.1 \mathrm{Mb})]$, or [gnuzip-ed postscript $(850 \mathrm{~Kb})]$.

Available: https://brainweb.bic.mni.mcgill.ca/brainweb/

[14]. R.K.-S. Kwan, A.C. Evans, G.B. Pike : "MRI simulation-based evaluation of image-processing and classification methods" IEEE Transactions on Medical Imaging. 18(11):1085-97, Nov 1999. Available: https://brainweb.bic.mni.mcgill.ca/brainweb/

[15]. D.L. Collins, A.P. Zijdenbos, V. Kollokian, J.G. Sled, N.J. Kabani, C.J. Holmes, A.C. Evans : "Design and Construction of a Realistic Digital Brain Phantom" IEEE Transactions on Medical Imaging, vol.17, No.3, p.463--468, June 1998. paper available in [html].

[16]. R.K.-S. Kwan, A.C. Evans, G.B. Pike : "An Extensible MRI Simulator for Post-Processing Evaluation" Visualization in Biomedical Computing (VBC'96). Lecture Notes in Computer Science, vol. 1131. SpringerVerlag, 1996. 135-140. paper available in [html], [postscript (1Mb)], or [gnuzip-ed postscript $(380 \mathrm{~Kb})]$. poster available in [grey-scale postscript $(5.3 \mathrm{Mb})$ ], [grey-scale, gnuzip-ed postscript $(536 \mathrm{~Kb})]$, or [colour, gnuziped postscript $(597 \mathrm{~Kb})]$.

[17]. Ewaryst, Rafajłowicz: "Data Structures for Pattern and Image Recognition and Application to Quality Control", Acta Polytechnica Hungarica, 2018, 15(4). DoI: 10.12700/APH.15.4.2018.4.13

[18]. Jiawei Lai, Hongqing Zhu, Xiaofeng Ling,: "Segmentation of Brain MR Images by Using Fully Convolutional Network and Gaussian Mixture Model with Spatial Constraints" 2019, Hindawi, Mathematical Problems in Engineering, Vol. 2019, Article ID 4625371, DOI: $10.1155 / 2019 / 4625371$

[19]. Yang J, Fan J, Ai D, Zhou S, Tang S, Wang Y. Brain MR image denoising for Rician noise using pre-smooth non-local means filter. Biomed Eng Online. 2015;14:2. Published 2015 Jan 9. DoI: 10.1186/1475-925X-14-2

[20]. Y. Zhou and H. Zhu, "Image segmentation using a trimmed likelihood estimator in the asymmetric mixture model based on generalized gamma and gaussian distributions", Mathematical Problems in Engineering, vol. 2018, Article ID 3468967, 17 pages, 2018. DoI: 10.1155/2018/3468967

[21]. Rupali. S. K., Kalyani R. J.,: “A Robust Optimized Feature Set Based Automatic Classification Of Alzheimer's Disease From Brain Mr Images Using K-Nn And Adaboost", ICTACT Journal On Image And Video Processing, 2017, Vol. 08, ISSUE: 03, ISSN: 0976-9102 (ONLINE), Dor: $10.21917 /$ ijivp.2017.0234

[22]. C. Nikou, A. C. Likas, and N. P. Galatsanos, “A Bayesian framework for image segmentation with spatially varying mixtures," IEEE Transactions on Image Processing, vol. 19, no. 9, pp. 2278-2289, 2010. DoI: $10.1109 /$ TIP.2010.2047903
[23]. Zhang X, Hou G, Ma J, Yang W, Lin B, et al.: "Denoising MR Images Using Non-Local Means Filter with Combined Patch and Pixel Similarity", 2014, PLOS ONE 9(6): e100240.

DoI: 10.1371/journal.pone. 0100240

[24]. I. Horváth, A. Sudár: "Factors Contributing to the Enhanced Performance of the MaxWhere 3D VR Platform in the Distribution of Digital Information", in Acta Polytechnica Hungarica, vol 15, no 3. 2018. DOI: 10.12700/APH.15.3.2018.3.9

[25]. V. Rentoumi et al., "Automatic detection of linguistic indicators as a means of early detection of Alzheimer's disease and of related dementias: A computational linguistics analysis," 2017 8th IEEE International Conference on Cognitive Infocommunications (CogInfoCom), Debrecen, 2017, pp. 000033-000038. DoI: 10.1109/CogInfoCom.2017.8268212

[26]. Mohammad Moghadasi, Seyed Majid Mousavi and Gábor Fazekas, "Cloud Computing Auditing", International Journal of Advanced Computer Science and Applications(IJACSA), 9(12), 2018. DoI: 10.14569/IJACSA.2018.091265

[27]. Ciccarelli, O., Thompson, A. "Managing the complexity of multiple sclerosis", Nature Reviews Neurology, 12, pp. 70-72, 2016, DOI: 10.1038/nrneurol.2016.2

[28]. Lötsch, J., Schiffmann, S., Schmitz, K. et al. "Machine-learning based lipid mediator serum concentration patterns allow identification of multiple sclerosis patients with high accuracy", Sci Rep 8, 14884 (2018). DoI: $10.1038 / \mathrm{s} 41598-018-33077-8$

[29]. A. D. Kovács and Z. Kvasznicza, "Use of 3D VR environment for educational administration efficiency purposes," 2018 9th IEEE International Conference on Cognitive Infocommunications (CogInfoCom), Budapest, Hungary, 2018, pp. 000361-000366. Dor: 10.1109/CogInfoCom.2018.8639964

[30]. Vér, Csaba: "3D VR spaces support R\&D project management.” 2018 9th IEEE International Conference on Cognitive Infocommunications (CogInfoCom), Budapest, 2018: 000375-000378. DOI: $10.1109 /$ coginfocom.2018.8639900

[31]. Berki,Borbála.: "2DAdvertising in 3D Virtual Spaces",Acta Polytechnica Hungarica, 2018, 15(3). DoI: 10.12700/APH.15.3.2018.3.10

[32]. Aaron Carass,et al.: "Longitudinal multiple sclerosis lesion segmentation: Resource and challenge", NeuroImage, Vol. 148, 2017, pp. 77-102, ISSN: 1053-8119, DOI: 10.1016/j.neuroimage.2016.12.064.

[33]. G. Csapó: "Sprego virtual collaboration space: Improvement guidelines for the MaxWhere Seminar system", 2017 8th IEEE International Conference on Cognitive Infocommunications (CogInfoCom), Debrecen, 2017, DoI: 10.1109/CogInfoCom.2017.8268231

[34]. Csapó, Gábor \& Csernoch, Maria \& Abari, Kálmán., "Sprego: case study on the effectiveness of teaching spreadsheet management with schema construction. Education and Information Technologies", Education and Information Technologies, 2019, DOI: 10.1007/s10639-019-10024-2

[35]. J. Schmidhuber., "Deep Learning in Neural Networks: An Overview", Neural Networks, Vol. 61, 2015, pp. 85-117. DoI: $10.1016 /$ j.neunet.2014.09.003

[36]. I. Horváth, "Disruptive technologies in higher education", 2016 7th IEEE International Conference on Cognitive Infocommunications (CogInfoCom), Wroclaw, 2016, pp. 000347-000352. DoI: 10.1109/CogInfoCom.2016.7804574

[37]. János Tóth, Róbert Tornai, Imre Labancz, András Hajdu: "Efficient Visualization for an Ensemble-based System", Special Issue on Cognitive Infocommunications (2019): Acta Polytechnica Hungarica Vol. 16, No. 2, 2019, DoI: 10.12700/APH.16.2.2019.2.4

[38]. Komlosi, Laszlo Imre and Patrick Waldbuesser. "The cognitive entity generation: Emergent properties in social cognition." 2015 6th IEEE International Conference on Cognitive Infocommunications (CogInfoCom), Gyor, 2015, pp.439-442. DoI: $10.1109 / \operatorname{Cog}$ InfoCom.2015.7390633 
Multiple sclerosis Lesion Detection via Machine Learning Algorithm

based on converting $3 \mathrm{D}$ to $2 \mathrm{D} \mathrm{MRI}$ images

[39]. Jovan Galic, Branislav Popovic, Dragana Sumarac Pavlovic: "Whispered Speech Recognition using Hidden Markov Models and Support Vector Machines", Special Issue on Cognitive Infocommunications (2018): Acta Polytechnica Hungarica Vol. 15, No. 5, 2018, DoI: 10.12700/APH.15.5.2018.5.2

[40]. S. M. Mousavi, M. Moghadasi and G. Fazekas, "Dynamic resource allocation using combinatorial methods in Cloud: A case study," 2017 8th IEEE International Conference on Cognitive Infocommunications (CogInfoCom), Debrecen, 2017, pp. 000073-000078. DoI: $10.1109 /$ CogInfoCom.2017.8268219

[41]. Sápi, J. Kovács, L. Drexler, D. Kocsis, P. Gajári, D. Sápi, Z., "Tumor Volume Estimation and Quasi-Continuous Administration for Most Effective Bevacizumab Therapy", 2015, PloS one. 10. e0142190. DoI: 10.1371/journal.pone.0142190

[42]. M. Moghadasi, G. Fazekas: "Multiple Sclerosis Detection via Machine Learning Algorithm, Accurate Simulated Database 3D MRI to 2D Images, using value of Binary Pattern Classification - A Case Study", 2019 10th IEEE International Conference on Cognitive Infocommunications (CogInfoCom), Naples, 2019, pp. 000233-000240.

[43]. Z. Karimaghaloo, M. Shah, S. J. Francis, D. L. Arnold, D. L. Collins and T. Arbel, "Automatic Detection of Gadolinium-Enhancing Multiple Sclerosis Lesions in Brain MRI Using Conditional Random Fields," in IEEE Transactions on Medical Imaging, vol. 31, no. 6, pp. 1181-1194, June 2012. DoI: 10.1109/TMI.2012.2186639

[44]. L. Kovács, Gy. Eigner: "Tensor Product Model Transformation-based Parallel Distributed Control of Tumor Growth", Volume 15, Issue Number 3, 2018. DoI: 10.12700/APH.15.3.2018.3.7

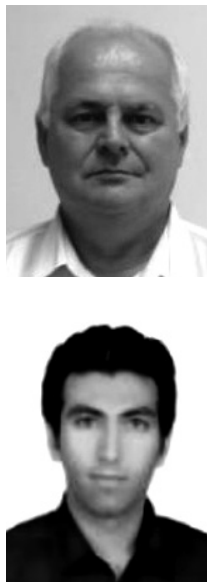

Dr. Gabor Fazekas is an Associate Professor at University of Debrecen, Faculty of Informatics, Department of Information Technology. He has extensive experience in supervising $\mathrm{PhD}$ dissertations. $\mathrm{He}$ is very well knowledged in reeasrch and teaching. $\mathrm{He}$ recently was retired but still supervising $\mathrm{PhD}$ students in the fields of computer systems, databases, image processing, combinatorial coding, didactic and many more.

Moghadasi Mohammad is a $\mathrm{PhD}$ student at University of Debrecen, Faculty of Informatics, with a backgorund of Software Information Technology. $\mathrm{He}$ is currently working in digital image processing on multiple sclerosis lesion detection. He is interested and has experience in database, networking and cloud computing as well. He is also a lecturer.
X.ApPENDiX A

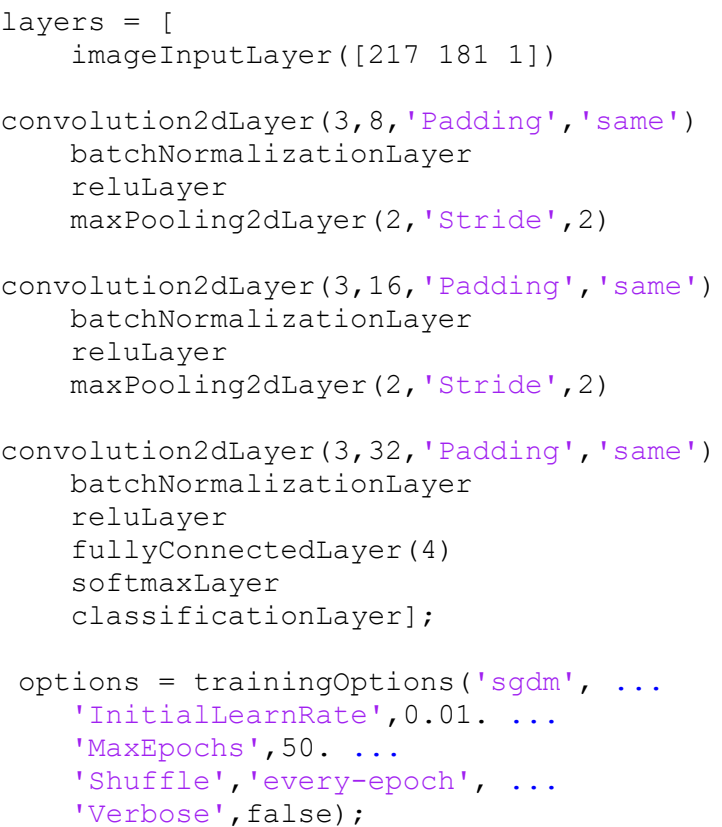

